\title{
Performance of the Analog Moving Window Detector
}

\author{
Hansen, V. Gregers
}

Published in:

I E E E Transactions on Aerospace and Electronic Systems

Link to article, DOI:

10.1109/TAES.1970.310099

Publication date:

1970

Document Version

Publisher's PDF, also known as Version of record

Link back to DTU Orbit

Citation (APA):

Hansen, V. G. (1970). Performance of the Analog Moving Window Detector. I E E E Transactions on Aerospace and Electronic Systems, 6(2), 173-179. https://doi.org/10.1109/TAES.1970.310099

\section{General rights}

Copyright and moral rights for the publications made accessible in the public portal are retained by the authors and/or other copyright owners and it is a condition of accessing publications that users recognise and abide by the legal requirements associated with these rights.

- Users may download and print one copy of any publication from the public portal for the purpose of private study or research.

- You may not further distribute the material or use it for any profit-making activity or commercial gain

- You may freely distribute the URL identifying the publication in the public portal

If you believe that this document breaches copyright please contact us providing details, and we will remove access to the work immediately and investigate your claim 


\section{Performance of the Analog Moving Window Detector}

\author{
V. GREgers hanSEN, Member, IEEE \\ Royal Technical University \\ of Denmark \\ Lyngby, Denmark
}

\begin{abstract}
A type of analog integrating moving window detector for use with a scanning pulse radar is examined. A performance analysis is carried out, which takes into account both the radiation pattern of the antenna and the dynamic character of the detection process due to the angular scanning of the antenna. An expression for the false alarm rate of the detector is first derived and evaluated numerically. The detection performance and angular accuracy are next determined in a direct Monte Carlo simulation of the detector on a digital computer for both no fading and pulse-to-pulse Rayleigh fading. Finally the influence on detection performance of the width used for the moving window is investigated.
\end{abstract}

Manuscript received June 9, 1969.

The author is now with the Raytheon Company, Wayland, Mass.

\section{Introduction}

The detection of radar signals in Gaussian noise by methods of analog post-detection integration has been analyzed in the past for a number of pertinent cases [1][5]. The differences among these have been with regard to assumptions concerning the envelope detector law, the antenna beam shape, and weighting in the integration as well as the chosen method of analysis.

All of these papers have, on the other hand, been based on the one-point theory of detection [6], which assumes that the statistical test for target presence can be carried out with exact knowledge of the target position.

In the present paper an analysis of a type of analog integrating moving window radar detector (abbreviated AMW detector for analog moving window detector) is carried out. This does not assume the target azimuth to be known and therefore is not restricted to the one-point theory. The analysis is however based on the assumption of a known target range such that the usual approximations would have to be used to derive the performance for an extended range interval.

The AMW detector is similar in principle to the wellknown binary digital moving window detector and essentially consists of a number of delay lines, each equal in length to the interpulse period, such that a running sum of the last $L$ video amplitudes can be formed on each sweep as a function of range. Based on certain dynamic detection criteria, as described in Section II, the false alarm rate and the detection performance of this detector are determined at a given range. For the detection performance, which is found by Monte Carlo simulation, a Gaussian antenna pattern is assumed and both no fading and pulseto-pulse Rayleigh fading are considered. Further, some results on angular accuracy are presented for a beamsplitting azimuth estimation logic.

\section{An Analog Moving Window Detector}

The AMW detector considered here is similar to the digital binary moving window integrator. However, in place of quantizing the incoming video in amplitude, it operates directly with the analog information. A block diagram of the complete receiver is shown in Fig. 1 and an input consisting of signals and additive stationary white Gaussian noise is assumed.

If the amplitude of the radar video signal at range $R$ and in the $i$ th sweep is denoted $x(R, i)$, the output of the AMW detector at range $R$ and in the $k$ th sweep is

$$
y(R, k)=\sum_{i=k-L+1}^{k} x(R, i)
$$

where $L$ represents the window width of the integrator. The output is thus the running sum of the received video amplitudes over the previous $L$ sweeps. The output can also be defined by the recurrence relation

$$
y(R, k)=y(R, k-1)-x(R, k-L)+x(R, k) .
$$




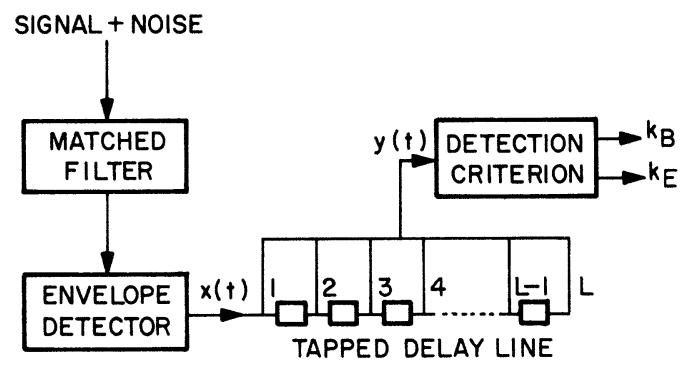

Fig. 1. Block diagram of radar receiver using analog moving window detector.

\section{False Alarm Performance}

The probability of obtaining a false alarm on the $k$ th sweep and at range $R$ is determined as the probability of occurrence of the target detection event specified by (3) for the case where only noise is present. ${ }^{3}$ We have

$$
P_{f s}=\operatorname{Prob}\left\{y(R, k) \geq V_{T}, y(R, k-1)<V_{T}\right\}
$$

where the second subscript in $P_{f s}$ indicates that this probability applies independently for each single sweep. By defining

$$
z(R, k)=\sum_{i=k-L+2}^{k} x(R, i)
$$

On the basis of the integrated output as given by (1) or (2) and a threshold $V_{T}$, the detection of a new target on the $k$ th sweep and at range $R$ is defined by the combined event $^{1}$

$$
y(R, k) \geq V_{T}
$$

and

$$
y(R, k-1)<V_{T}
$$

we can write

$$
y(R, k)=z(R, k-1)+x(R, k)
$$

and

$$
y(R, k-1)=z(R, k-1)+x(R, k-L)
$$

and the false alarm probability can equivalently be stated as

$$
\begin{aligned}
P_{f s}= & \operatorname{Prob}\left\{x(R, k) \geq V_{T}-z(R, k-1),\right. \\
& \left.x(R, k-L)<V_{T}-z(R, k-1)\right\} .
\end{aligned}
$$

Assuming all $x(R, i)$ statistically independent ${ }^{4}$ and identically distributed it follows that $x(R, k), x(R, k-L)$, and $z(R, k-1)$ all will be statistically independent and the false alarm probability is determined as

$$
\begin{aligned}
P_{f \boldsymbol{z}}= & \int_{0}^{V_{T}} p_{L-1}(z) \\
& \cdot\left[\int_{0}^{V_{T}-z} p_{1}(x) d x \int_{V_{T}-z}^{\infty} p_{1}(x) d x\right] d z
\end{aligned}
$$

where the arguments of $x(R, i)$ and $z(R, k)$ have been omitted for simplicity so that $x=x(R, i)$ and $z=z(R, k)$. The probability density function for $x(R, i)$ is denoted $p_{1}(x)$ and for $z(R, k-1)$ it is $p_{L-1}(z)$. Since $z(R, k-1)$ is the sum of $L-1$ independent variables (9) each having the pdf $p_{1}(x)$ we have

$$
\begin{aligned}
p_{L-1}(z)= & \left.p_{1}(x) * p_{1}(x) * \cdots * p_{1}(x)\right|_{x=z} \\
& \longleftarrow-1 \text { times } \longrightarrow
\end{aligned}
$$

where the asterisk denotes the convolution operation.

Assuming a linear envelope detector after the matched filter and normalizing the power level at the filter output to unity, the video amplitude $x(R, i)$ will have a Rayleigh pdf when only noise is present, i.e.,

$$
p_{1}(x)=x \exp \left(-x^{2} / 2\right) \quad x \geq 0 .
$$

Inserting into (13) then gives the false alarm probability

\footnotetext{
${ }^{1}$ Usually the range interval of interest will be quantized into a number of contiguous range bins such that $R$ should be considered as a discrete parameter in (3).

2 The effect of an additional criterion which requires that a new target detection must have a certain angular separation from a previous detection is investigated in Section III.
}

${ }^{3}$ The derivation is similar to that presented in [7] which, however, is for a binary moving window detector.

${ }^{4}$ This may, for example, not be true if the interference is due to distributed clutter returns. 


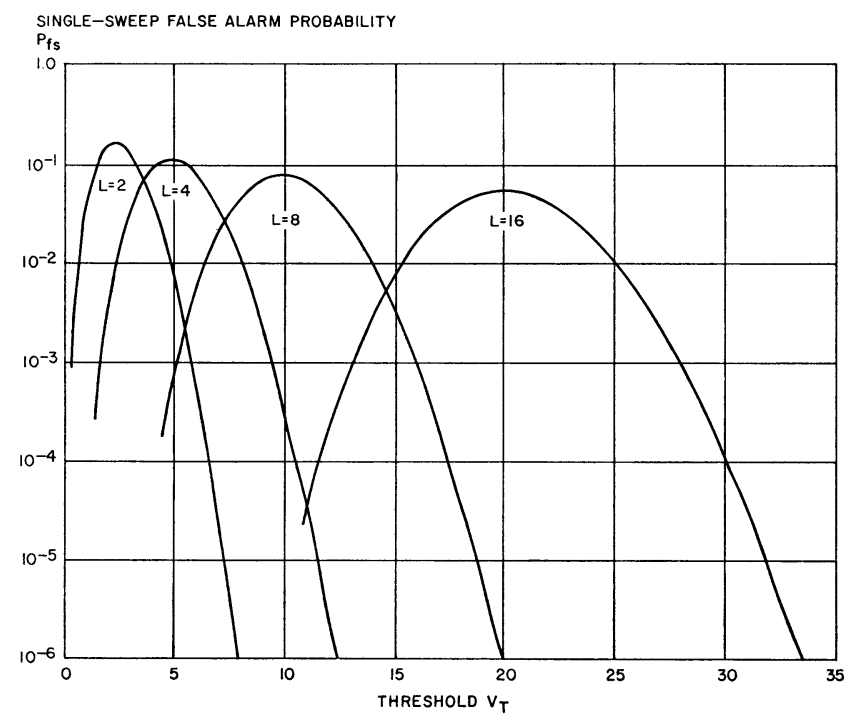

Fig. 2. Single-sweep false alarm probability $P_{f s}$ versus threshold for AMW detector.

$$
\begin{aligned}
P_{f s}= & \int_{0}^{V T} p_{L-1}(z)\left[\left(1-\exp \left(-\left(V_{T}-z\right)^{2} / 2\right)\right)\right. \\
& \left.\cdot \exp \left(-\left(V_{T}-z\right)^{2} / 2\right)\right] d z .
\end{aligned}
$$

This equation was evaluated numerically for various values of $L$ by first determining $p_{L-1}(z)$ as the $(L-1)$-fold self-convolution of $p_{1}(x)$ and then carrying out the integration in (16). In the convolutions as well as in the final integral a discrete representation of the Rayleigh density function using $\Delta x=0.1$ was used. The results obtained are shown in Fig. 2 with $P_{f s}$ as a function of $V_{T}$ for $L=2$, 4,8 , and 16 . It is noted that the curves have a maximum value with $P_{f s}$ decreasing to both sides. The reason for this behavior is that for low values of the threshold the integrated output will stay above the threshold most of the time and positive threshold crossings (false alarms) will seldom occur.

From the results in Fig. 2 the false alarm rate (FAR) at a given range is determined as

$$
\mathrm{FAR}=P_{f s} f_{R} \quad \text { alarms per second, }
$$

where $f_{R}$ is the radar pulse repetition frequency. In order to obtain the overall false alarm rate when an extended range interval is considered, approximate methods, based on the actual procedure used for quantizing range, would have to be introduced.

In the preceding analysis it was not taken into account that in a practical system a certain angular separation (0.5-1.0 beamwidth) will usually be required between two detections if they are to be considered as two distinct targets. To introduce this additional criterion into the above analysis appears to be difficult and its significance was therefore investigated through a direct computer simulation in which a sequence of video amplitudes $x(R, i), i=1,2, \cdots$, were generated as

\begin{tabular}{|c|c|c|c|c|}
\hline$L$ & $N_{F A}{ }^{*}$ & $\hat{P}_{f_{s}} \dagger$ & $N_{F D} \ddagger$ & $\begin{array}{c}\gamma_{F A} \| \\
\text { (percent) }\end{array}$ \\
\hline $\begin{array}{r}4 \\
8 \\
16\end{array}$ & $\begin{array}{l}50 \\
54 \\
56\end{array}$ & $\begin{array}{ll}1.0 & 10^{-3} \\
1.1 & 10^{-3} \\
1.1 & 10^{-3}\end{array}$ & $\begin{array}{r}1 \\
6 \\
13\end{array}$ & $\begin{array}{l}98 \\
89 \\
77\end{array}$ \\
\hline
\end{tabular}

TABLE I

Simulation over 50000 sweeps for $P_{f_{s}}=10^{-3}$

${ }^{*} N_{F A}$ is the total number of false alarms which occurred in simulation.

$\dagger \hat{P}_{f s}$ is the estimated false alarm probability $\left(=N_{F A} / 50000\right)$.

$\ddagger N_{F D}$ is the number of false alarms which were rejected by the separation criterion as specified above.

$\| \gamma_{F A}$ expresses the reduction in false alarm rate if a "onebeamwidth separation" criterion is used by the detector $\left(\gamma_{F A}=100\left(N_{F A}-N_{F D}\right) / N_{F A}\right)$.

$$
x(R, i)=\sqrt{{n_{1}{ }^{2}+n_{2}{ }^{2}}^{2}}
$$

where $n_{1}$ and $n_{2}$ are zero-mean unit-variance Gaussian random variables as obtained from a pseudorandom number generator in the computer. The window width $L$ was assumed to equal one beamwidth and this was also used as the separation criterion in the detector. The azimuth estimate according to (6) determines the angular position of a detected target and if two consecutive detections yield estimates $\hat{k}_{T 1}$ and $\hat{k}_{T 2}$, the latter detection is rejected according to the separation criterion if $\hat{k}_{T 2}-\hat{k}_{T 1} \leq L$.

The simulation was carried out for $L=4,8$, and 16 with decision thresholds giving $P_{f_{s}}=10^{-3}$ as obtained from Fig. 2. The results of a simulation over 50000 consecutive sweeps are summarized in Table $I$.

From these results it is seen that the additional criterion that detections must be separated by more than one beam- 


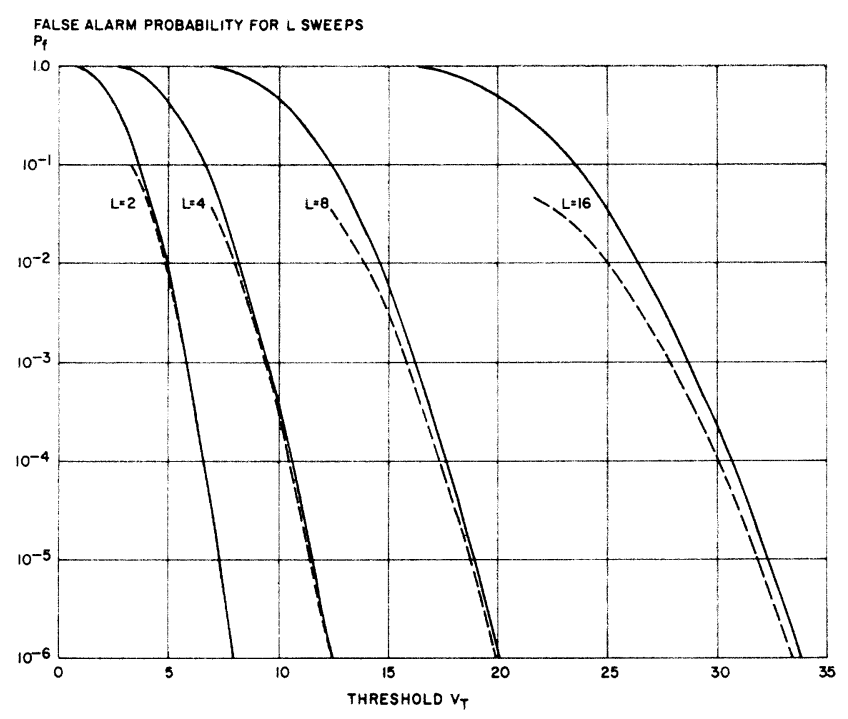

Fig. 3. False alarm probability $P_{f}$ versus threshold for analysis based on one-point theory. Curves shown in broken line are from Fig. 2.

width in order to be taken into account individually does reduce the effective false alarm rate and more so for the larger values of $L$. However, even for $L=16$, which is the largest value considered here, the reduction is rather modest, corresponding to no more than an equivalent change in signal-to-noise ratio of around $0.1 \mathrm{~dB}$.

For the detection model based on the one-point theory the false alarm probability would be determined as

$$
P_{f}=\int_{V_{T}}^{\infty} p_{L}(u) d u
$$

where $u$ is the sum of $L$ independent variables, each with the pdf $p_{1}(x)$, such that

$$
\begin{aligned}
& p_{L}(u)=\left.p_{1}(x) * p_{1}(x) * \cdots * p_{1}(x)\right|_{x=u} . \\
& \longleftarrow \quad L \text { times } \longrightarrow
\end{aligned}
$$

Equations (19) and (20) were evaluated numerically and the curves thus found are shown in Fig. 3 together with the results from Fig. 2 in broken line.

From these two sets of curves it is possible to determine the average duration of a false alarm with the moving window analog integrator, for a given value of the threshold $V_{T}$. The average duration of a false alarm is given by

$$
D_{\mathrm{av}}=E\left[k_{E}-k_{B}\right]=P_{f} / P_{f s} \geq 1 \text {. }
$$

The validity of (21) follows because $P_{f}$ is the probability that the integrated video is above the threshold $V_{T}$ for a randomly selected observation of $L$ consecutive video amplitudes in a long sequence, whereas $P_{f s}$ is the probability that such an observation corresponds to the target begin as defined by (3). Since the target begin condition is a subset of all observations where the integrated video exceeds the threshold the last inequality holds.

\section{Defection Curves and Angular Accuracy}

The detection performance for the AMW detector was next determined by a direct Monte Carlo simulation assuming a Gaussian characteristic for the antenna radiation pattern and a matched filter receiver as shown in Fig. 1. The number of signal pulses received between the $3 \mathrm{~dB}$ points of the antenna (one-way pattern) is denoted $M$ and is usually referred to as the number of hits per beamwidth.

For each repetition a sequence of more than $3 M$ video amplitudes was generated. Assuming a known target range each amplitude in the observed sequence was determined as

$$
x_{i}=\sqrt{\left(A_{i}+n_{1}\right)^{2}+n_{2}^{2}}
$$

where $n_{1}$ and $n_{2}$ are independent zero-mean Gaussian random variables with variance one, and $A_{i}$ is the signal amplitude in the $i$ th sweep as determined by the signalto-noise ratio $E / N_{0}$ at the center of the antenna beam, ${ }^{5}$ the relative antenna gain $G_{i}$ (two-way voltage gain) at the actual azimuth angle, and the target fading characteristics. Since the average power signal-to-noise ratio at the matched filter output equals the ratio of average signal energy to noise spectral density at the input, we have in the no fading case [due to the normalization of the noise assumed in (22)] that $\frac{1}{2} A_{1}{ }^{2}=G_{i}{ }^{2} E / N_{0}$ and thus the signal amplitude is determined as

$$
A_{i}=\sqrt{2 E / N_{0}} \cdot G_{i}
$$

${ }^{5} E$ is the average energy of a received signal pulse at the center of the beam and $N_{0}$ is the (one-sided) spectral density of the white Gaussian noise. 
PROBABILITY OF DETECTION

$P_{d}$

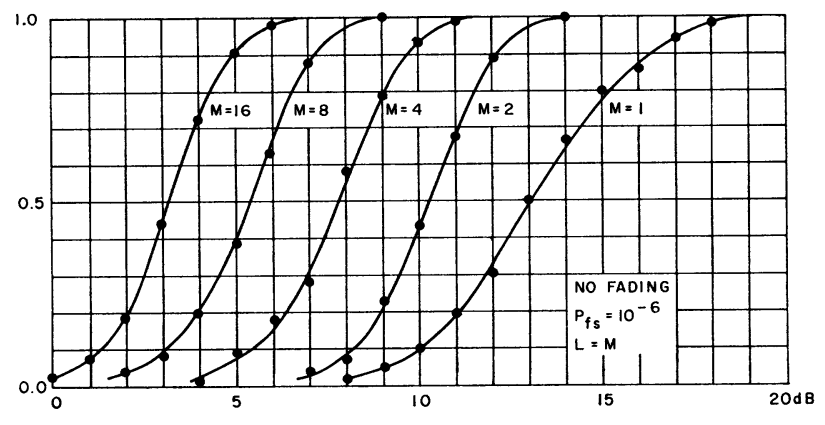

SINGLE-PULSE SIGNAL-TO-NOISE RATIO E/NO IN dB

Fig. 4. Detection performance of the $A M W$ detector for the no fading case. The points shown are the results of the simulation and the curves are drawn to fit these.

Fig. 5. Detection performance of the AMW detector for the case of pulse-to-pulse Rayleigh fading. The points sho $w^{n}$ are the results of the simulation and the curves are drawn to fit these.

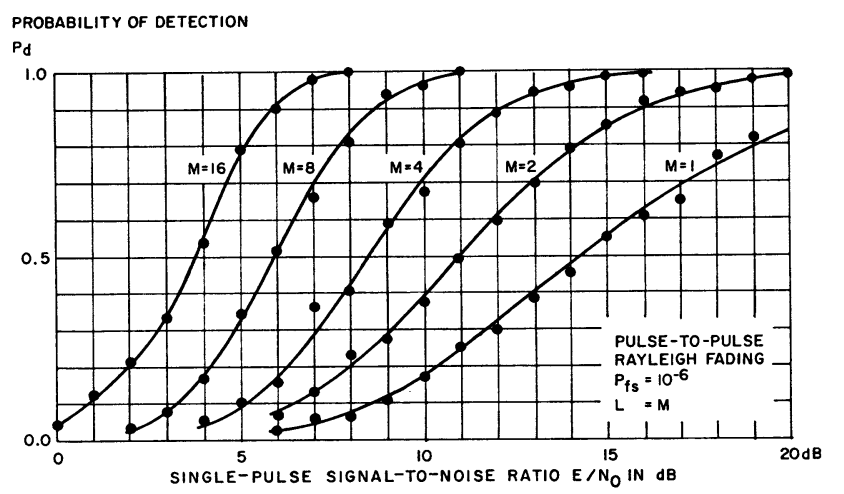

For a pulse-to-pulse Rayleigh fading amplitude we have

$$
\frac{1}{2} E\left[A_{i}{ }^{2}\right]=G_{i}{ }^{2} E / N_{0}
$$

which is satisfied when the Rayleigh amplitude is determined as

$$
A_{i}=\sqrt{a_{1}^{2}+a_{2}^{2}}
$$

where $a_{1}$ and $a_{2}$ are zero-mean Gaussian random variables with variance $G_{i}{ }^{2} \cdot E / N_{0}$.

The relative position of the antenna pattern and the individual returns was randomized over one sweep-tosweep period at the start of each new video sequence. Each video sequence was in turn examined by the moving window analog integrator as specified by (2) for different values of the window width $L$ and using the curves of Fig. 2 to determine the thresholds for which $P_{f_{s}}=10^{-6}$. For each repetition the occurrence of a target detection was recorded together with the corresponding azimuth estimate $\hat{k}$ as determined by the beam-splitting logic specified by (6).

After the desired number of repetitions $N_{r}$, the detection probability was estimated as the ratio between the number of actual detections and $N_{r}$, and the sample mean and sample variance of the azimuth estimate were determined.

The results of the simulation showing the detection probability $P_{d}$ versus the reference signal-to-noise ratio $E / N_{0}$, obtained for $M=1,2,4,8$, and 16 and taking $L=M$, are shown in Fig. 4 for the no fading case and in Fig. 5 for pulse-to-pulse Rayleigh fading. For $M=1$ and 2 the number of repetitions was $N_{r}=500$ whereas for the other values of $M$ it was taken as $N_{r}=300$ in order to limit computation time. Similar results were simultaneously obtained for other values of $L$ by examining the video sequence generated in the simulation by the appropriate detector logics. These results are summarized in Fig. 6 and Fig. 7 for $P_{d}=0.5$ and $P_{f s}=10^{-6}$ by showing the required signal-to-noise ratio as a function of $L$ for different values of $M$. From these curves it is seen that the minimum required signal-to-noise ratios are obtained for $L \simeq M$. The minimum, however, is rather broad such that values of $L$ smaller than $M$ may be used with little degradation in performance.

Results obtained on angular accuracy are shown in Figs. 8 and 9 for the case where $L=M$. The estimated standard deviation (square root of sample variance) with the beam-splitting procedure is shown for $M=1,4$, and 16 and the lower bounds derived by Swerling [8], for no fading and pulse-to-pulse fading, respectively, are shown in broken line for reference. It should be noted that these bounds are derived under the assumption that the number of hits per beamwidth is large and therefore do not include the quantization error that one would expect for few hits per beamwidth. Results obtained in [9] show that for $M=20$ the accuracy of the maximum likelihood procedure is around 20 percent larger than the lower bound. The beam-splitting procedure is seen to give results close to this for low signal-to-noise ratios but for increasing signal-to-noise ratio the standard deviation decreases at a rate much slower than the bounds. This is presumably because the threshold crossings corresponding to target begin and target end will always take place in regions corresponding to a marginal signal-to-noise ratio such that only the increased steepness of the antenna beam pattern further from the beam center accounts for the improved accuracy of the estimate for larger signal-to-noise ratios.

The odd shape of the curves for $M=1$ probably occurs because a marginal signal-to-noise ratio (and therefore a low detection probability) will make the detections tend to occur mainly when a target return coincides with the center of the antenna beam, thus improving angular accuracy.

The bias of the estimator was found equal to the theoretically expected value of $L / 2$. 
SIGNAL-TO-NOISE RATIO

$E / N_{O} d B$

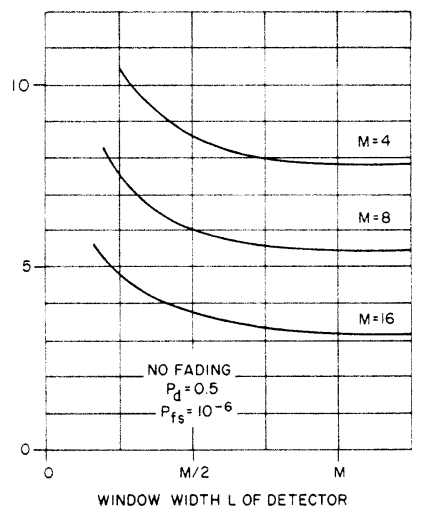

Fig. 6. Required signal-to-noise ratio for the AMW detector to yield $P_{d}=0.5$ and $P_{f s}=10^{-6}$ in the no fading case, as a function of the window width $L$.

Fig. 7. Required signal-to-noise ratio for the AMW detector to yield $P_{d}=0.5$ and $P_{f s}=10^{-6}$ in the case of pulse-to-pulse Rayleigh fading, as a function of the window width $L$.

SIGNAL-TO-NOISE RATIO

$E / N_{O} d B$

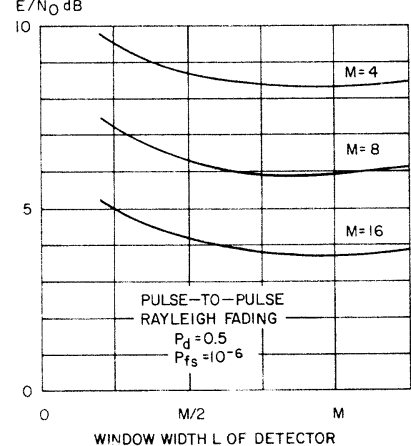

WINDOW WIDTH L OF DETECTOR
RELATIVE ANGULAR ACCURACY

$\sigma_{\theta} / \theta_{3 d B}$

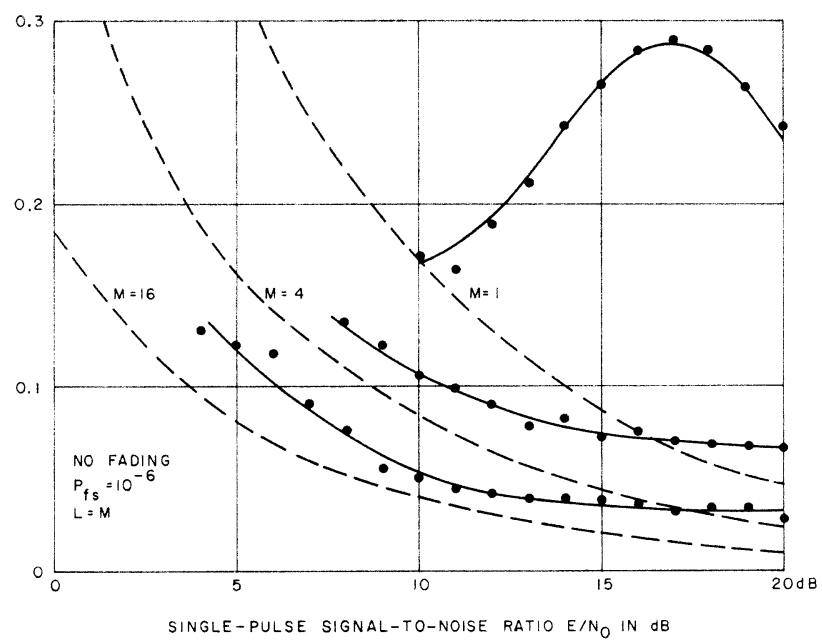

Fig. 8. Angular accuracy obtained with beam-splitting estimation procedure for the no fading case. Broken line curves are lower bounds derived by Swerling [8] and points shown are simulation results.

Fig. 9. Angular accuracy obtained with beam-splitting estimation procedure for pulse-to-pulse Rayleigh fading case. Broken line curves are lower bounds derived by Swerling [8] and points shown are simulation results.

RELATIVE ANGULAR ACCURACY $\sigma_{\theta} / \theta_{3 \mathrm{~dB}}$

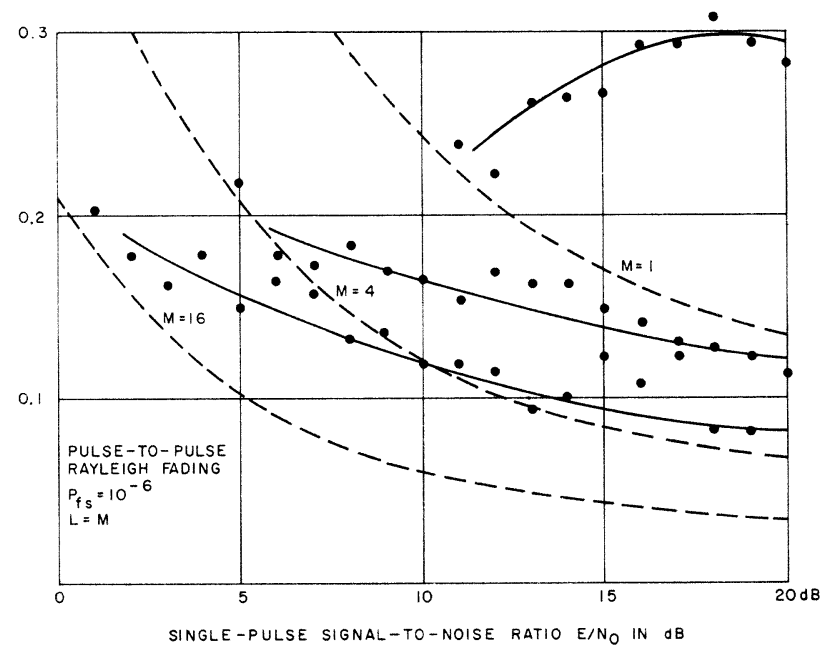

\section{Conclusions}

A type of analog integrating moving window detector has been analyzed, taking into account the antenna pattern and the dynamic character of the detection process. The performance of this detector is expected to be close to the maximum likelihood procedure and the results obtained should thus be useful for reference purposes. The maximum likelihood detector would be obtained by using the optimum envelope detector law, making the width of the window several times the beamwidth, and applying optimum weights to the outputs of the delay line. To determine the false alarm rate in this case, along the lines

of Section III of this paper, appears to be difficult, and a Monte Carlo analysis of the maximum likelihood detector, as in Section IV, could therefore not be carried out. Some results for optimum weighted integration based on the one-point theory have been obtained in [4] and [5] and may be taken as indicative of the difference in performance. In [5] the asymptotic improvement with optimum weighting as compared to uniform integration over the $3 \mathrm{~dB}$ beamwidth is found to be $0.23 \mathrm{~dB}$. For the same 
comparison an improvement of $0.7 \mathrm{~dB}$ is quoted in [4] for $M=50, P_{d}=0.5$, and $P_{f}=10^{-6}$. These two results are diffcult to reconcile but the method used in [4] is probably not very accurate.

The results on angular accuracy with the beam-splitting estimation procedure show that for low signal-tonoise ratio the performance is close to the maximum likelihood procedure. For increasing signal-to-noise ratio the accuracy does, however, not follow the lower bounds but decreases at a much slower rate. Whether or not such a behavior would be acceptable in practice would depend on the particular application.

\section{Acknowledgment}

The author is indebted to D. K. Barton for his helpful comments on the manuscript. Computer time was made available by the Northern Europe University Computing Center (NEUCC), Lyngby, Denmark, on its IBM 7094 installation.

\section{References}

[1] J. I. Marcum, "A statistical theory of target detection by pulsed radar," IRE Trans. Information Theory, vol. IT-6, pp. 59-144, April 1960.

[2] E. L. Kaplan, "Signal-detection studies with applications," Bell Sys. Tech. J., vol. 34, pp. 403-437, March 1955.

[3] D. S. Palmer and D. C. Cooper, "An analysis of the performance of weighted integrators," IEEE Trans. Information Theory, vol. IT-10, pp. 296-302, October 1964.

[4] V. A. Likharev, "Effectiveness of weighted summing of an echosignal packet in analog and digital processing," Radio Engineering, vol. 23, no. 6, pp. 119-121, 1968.

[5] W. M. Hall, "Antenna beam-shape factor in scanning radars," IEEE Trans. Aerospace and Electronic Systems, vol. AES-4, pp. 402-409, May 1968.

[6] J. L. Lawson and G. E. Uhlenbeck, Threshold Signals. New York: McGraw-Hill, 1950, p. 162.

[7] D. E. Hampton and A. A. McCurrach, "False alarm rates in a digital detector," Royal Aircraft Establishment, Tech. Note Rad. 789, October 1960.

[8] P. Swerling, "Maximum angular accuracy of a pulsed search radar," Proc. IRE, vol. 44, pp. 1146-1155, September 1956.

[9] V. V. Avdeyev and V. A. Likharev, "The effectiveness of maximum-likelihood direction-finding," Telecomm. Radio Engrg., pt. 2, vol. 22, no. 8, pp. 134-138, 1967.

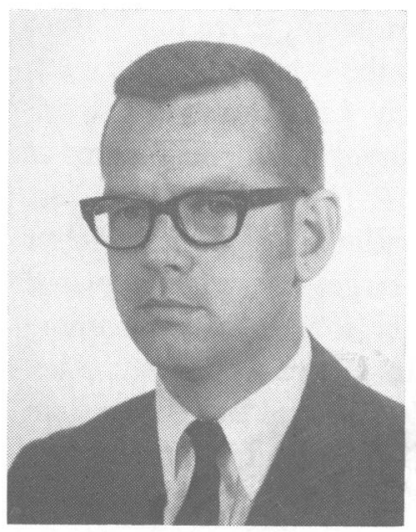

Vilhelm Gregers Hansen (M'60) was born in Odense, Denmark, on September 30, 1934. He received the M.Sc. degree in electrical engineering from the Royal Technical University of Denmark in 1959.

He served with the Danish Defense Research Board from 1959 to 1960, and from 1960 to 1966 he was employed with the SHAPE Technical Centre, The Hague, Netherlands, where he worked primarily on radar detection and anti-jamming techniques, and related evaluation methods. From 1966 to 1969 he was Assistant Professor at the Royal Technical University of Denmark, teaching courses in detection theory and communication theory and doing research on related subjects. He recently joined the Raytheon Company, Wayland, Mass., as a member of the Technical Staff of the Radar Systems Laboratory. 